\begin{tabular}{|c|c|}
\hline Title & Fundamental study on measurement of ELF electric field at biological body surfaces \\
\hline Author(s) & Shimizu, K.; Endo, H.; Matsumoto, G. \\
\hline Citation & $\begin{array}{l}\text { IEEE Transactions on Instrumentation and Measurement, 38(3), 779- } 784 \\
\text { https://doi.org/10.1109/19.32192 }\end{array}$ \\
\hline Issue Date & 1989-06 \\
\hline Doc URL & http:/hdl.handle.net/2115/6045 \\
\hline Rights & $\begin{array}{l}\text { (C1989 IEEE. Personal use of this material is permitted. However, permission to reprint/republish this material for } \\
\text { advertising or promotional purposes or for creating new collective works for resale or redistribution to servers or lists, } \\
\text { or to reuse any copyrighted component of this work in other works must be obtained from the IEEE. } \\
\text { IEEE, IEEE Transactions on Instrumentation and Measurement, 38(3), 1989, p779-784 }\end{array}$ \\
\hline Type & article \\
\hline File Information & ITIM38_3.pdf \\
\hline
\end{tabular}

Instructions for use 


\title{
Fundamental Study on Measurement of ELF Electric Field at Biological Body Surfaces
}

\author{
KOICHI SHIMIZU, MEMBER, IEEE, HIDETO ENDO, AND GORO MATSUMOTO, FELlOW, IEEE
}

\begin{abstract}
In the study of biological effects of extremely low frequency (ELF) electric fields, a few techniques were developed which enable us to measure the electric field at a biological body surface with practical accuracy and spatial resolution. They are: pressing down a field sensor against the body surface, using a thin flexible sensor, and covering the body surface with a conductive layer while leaving the point of measurement uncovered. The characteristics of these techniques were also examined with a view toward practical applications, such as the measurement of the electric field at a human surface located under high voltage transmission lines.
\end{abstract}

\section{INTRODUCTION}

$\mathrm{W}$ ITH THE rapid increase in the power of electric facilities and electric equipment in our environment, there has been a growing interest in the biological effects of extremely low frequency (ELF) electric fields. The safety assessment of high voltage transmission lines has been considered to be urgent and many studies have been conducted [1]-[3]. The World Health Organization (WHO) has published the health criteria for exposure to ELF electric fields [4]. However, many important points concerning the biological effects such as the threshold and the mechanism remain unknown. In order to investigate the biological effects and to set up safety standards, an accurate measurement of the electric field at a biological body surface is essential. However, there seem to be few techniques available which are suitable for the field measurement at the geometrically complex surface of a biological body. Most of the field meters currently available [5] are large relative to the size of the morphological irregularity of the biological body. This causes a perturbation of the field around the point of measurement and results in erroneous measurements. This restriction makes field measurements with high spatial resolution on body surfaces difficult. To solve these problems, some techniques are developed which give better results in the measurement of the ELF electric field at a biological body surface. This paper presents the principle of the newly

Manuscript received September 19, 1988; revised December 10, 1988. This work was supported in part by the Ministry of Education, Science, and Culture under Grant-in-Aid for Scientific Research 63602036.

$\mathrm{K}$. Shimizu is with the Department of Bioengineering, Faculty of Engineering, Hokkaido University, Sapporo, 060, Japan:

H. Endo is with Suzuye and Suzuye Patent Attomeys, Tokyo, $100 \mathrm{Ja}-$ pan.

G. Matsumoto is with the Hokkaido Institute of Technology, Sapporo, 006, Japan.

IEEE Log Number 8926911. developed techniques and the results of the evaluation of these techniques.

\section{Principle of Field Measurement}

A displacement current flows into a biological body in the ELF electric field. The current $I$ induced in the area $S$ of the body surface is given as

$$
I=\int_{S} 2 \pi f \epsilon E(s) d s
$$

where

$$
\begin{aligned}
& f \quad \text { frequency of the field (e.g., } 50 \mathrm{~Hz}), \\
& \epsilon \quad \text { permittivity of air }\left(8.85 \times 10^{-12} \mathrm{~F} / \mathrm{m}\right), \\
& E(s) \quad \text { surface field strength at the position } s .
\end{aligned}
$$

When $E(s)$ is constant over the area $S$, such as on the ground plane in a uniform electric field, the current is given by

$$
I=2 \pi f \epsilon S E .
$$

When an object is placed in the uniform field, the $E(s)$ is no longer constant. For example, the electric field $E$ at the surface of the half cylinder placed on the ground plane with the round face up (Fig. 1) is given by the following equation, e.g., and the induced current has to be calculated using the (1):

$$
E\left(\theta_{s}\right)=2 E_{0} \cos \theta_{s}
$$

where

$E_{0}$ unperturbed field,

$\theta_{s}$ circumferential angle shown in Fig. 1.

The length of the half cylinder in an axial direction is assumed to be infinite.

Conventionally the field measurement at an object surface has been performed using a disk-shape sensor which consists of two layers with a conductor and an insulator [6]. Fig. 2 shows a schematic view of the sensor with a guard-ring. Usually the guard-ring is kept at the same potential as the object and serves to eliminate the field concentration at the edge of the sensing part. Although the guard-ring is useful for this purpose, it inevitably makes the sensor large which degrades the spatial resolution and makes it unsuitable for the measurement at curved surfaces typical of biological bodies. Therefore, a few techniques which enable us to measure the electric field dis- 


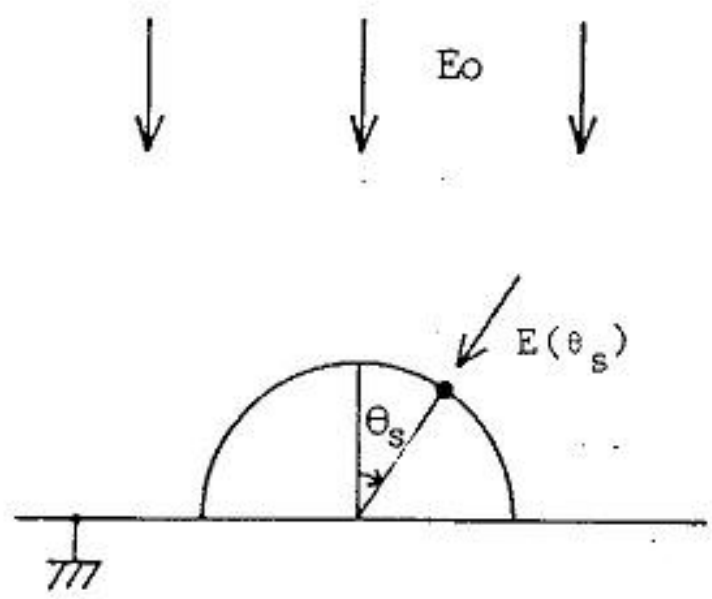

Fig. 1. Electric field at conductive half-cylinder surface placed on the ground plane. $\theta_{s}$ is a circumferential angle.

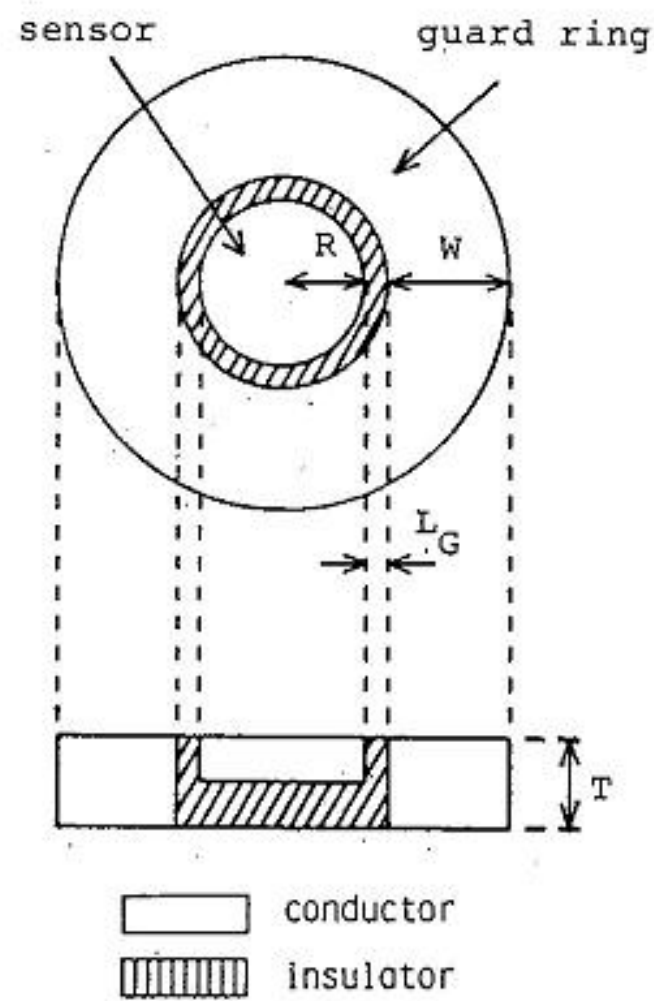

Fig. 2. Top and front (vertical cross section) views of the sensor with a guard-ring: $R=$ sensor radius, $W=$ guard-ring width, $T=$ sensor thickness, $L_{G}=$ sensor guard-ring spacing.

tribution at a biological body surface with reasonable accuracy and spatial resolution were developed. They are pressing down the sensor against the soft body surface, using a thin flexible sensor, and covering the body surface with a conductive layer leaving the point of measurement uncovered.

Fig. 3 shows schematic views of these methods for measurements at a ground plane. The induced current or the displacement current flows through the conductor and insulator of the sensor to the ground plane. The vertical lines in the right figures are lines of electric force. The direction of electric field is given as that of the tangent of the force lines. As can be seen in the case of the sensor on the ground (Fig. 3(a)), an unnecessary field concentration occurs around the edge of the sensor, and the obtained field value is larger than the true field. However, in the case of the sensor pressed on the soft surface (Fig. $3(b))$, the obtained field value is close to the true field, because the ground plane serves as the guard-ring. When the surrounding area is covered with a conductive layer (Fig. 3(c)), the field concentration occurs at the edge of the covering around the measurement parts, and the obtained value is less than the true field. Using both techniques shown in Fig. 3(a) and (c), the upper and lower limits of the field strength are given, and the true field strength can be estimated with a known error boundary. (a)
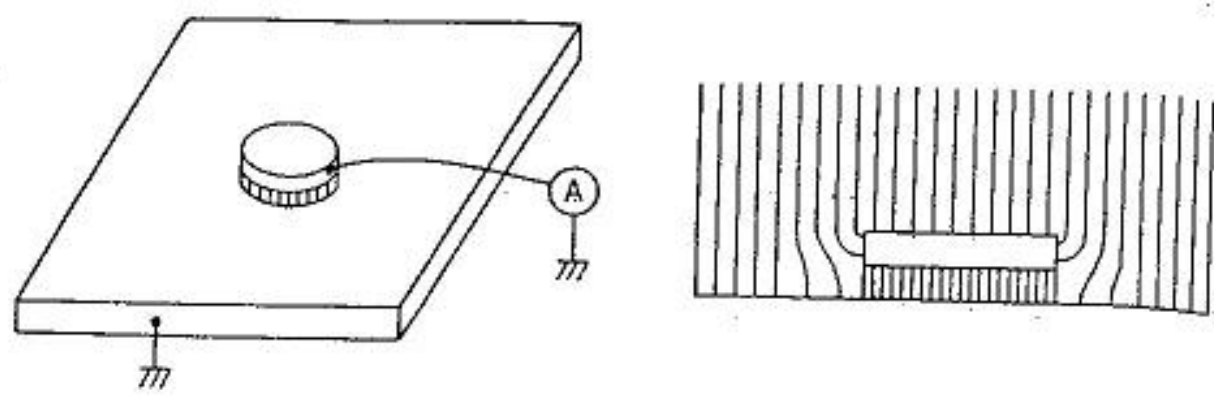

(b)
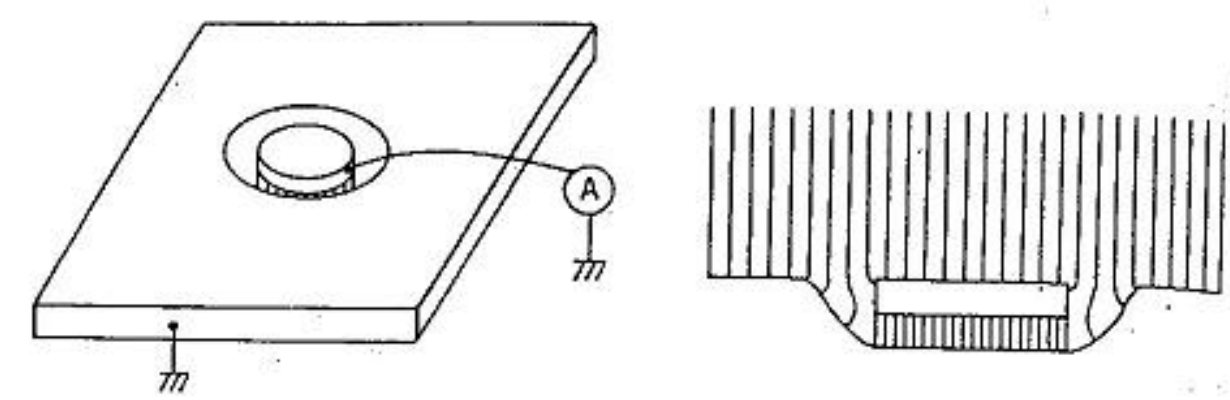

(c)
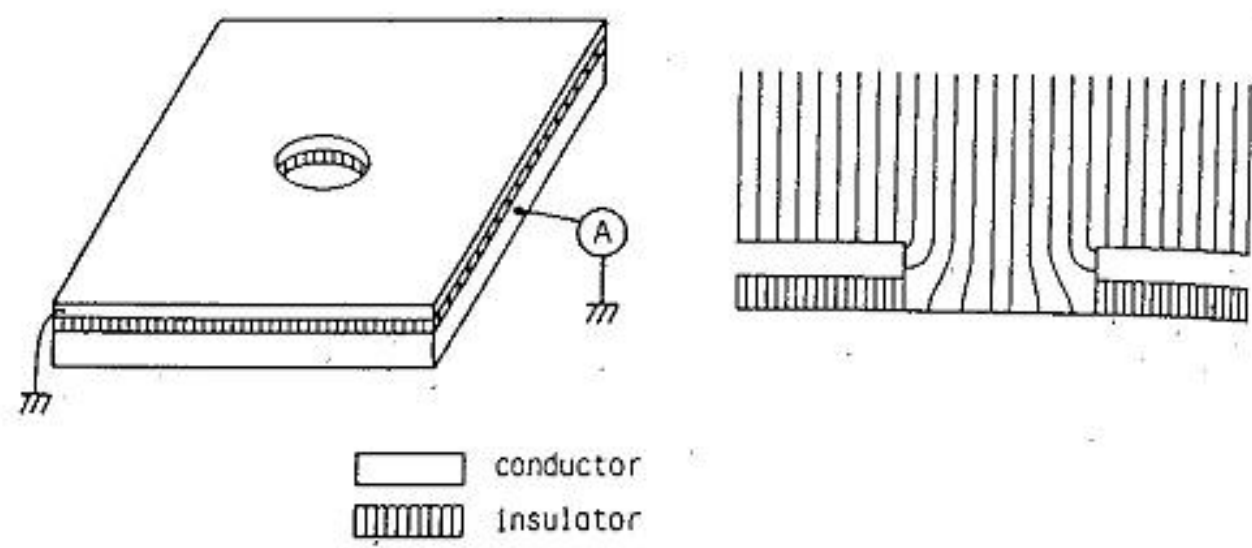

Fig. 3. Schematic views of three methods of field measurement. (a) With a sensor on the object. (b) With a pressed sensor. (c) With covering conductor. (Vertical lines in the right figures are electric lines of force.)

\section{METHHOD}

\section{A. Calculation}

In order to obtain the theoretical values of the electric field for the evaluation of the proposed techniques, the field at the sensor is calculated using a numerical calculation method such as the finite difference method [7]. In this method an object region is divided into a rectangular mesh of points, and the electrical potential of each point is obtained from the finite difference equation using the potentials of the surrounding points. The field was calculated for the case of a three dimensional axially symmetric field and with a regular grid configuration.

\section{B. Measurement}

In order to analyze the accuracy of the proposed techniques, the following experiments were performed. Fig. 4 shows schematic views of the experiments. The $50-\mathrm{Hz}$ unperturbed electric field $E_{0}(0-50 \mathrm{kV} / \mathrm{m})$ was generated between two circular plane parallel electrodes $(0.5 \mathrm{~m}$ in diameter) with $0.1-\mathrm{m}$ separation. In the experiments, the electric fields were measured in the different conditions of the sensor and compared against the theoretical values. The different conditions are as follows. 1) A sensor without a guard-ring was placed at the center of the lower electrode surface (Fig. 5(a)). 2) In order to simulate the field measurement at the soft surface of a biological body, the same sensor was pressed down on the lower electrode made of a soft conductive material (Fig. 5(b)). 3) A flexible sensor was placed along the curved surface of the half cylinder ( $3 \mathrm{~cm}$ in radius and $50 \mathrm{~cm}$ in length) which was placed on the lower electrode with the round face up (Fig. 

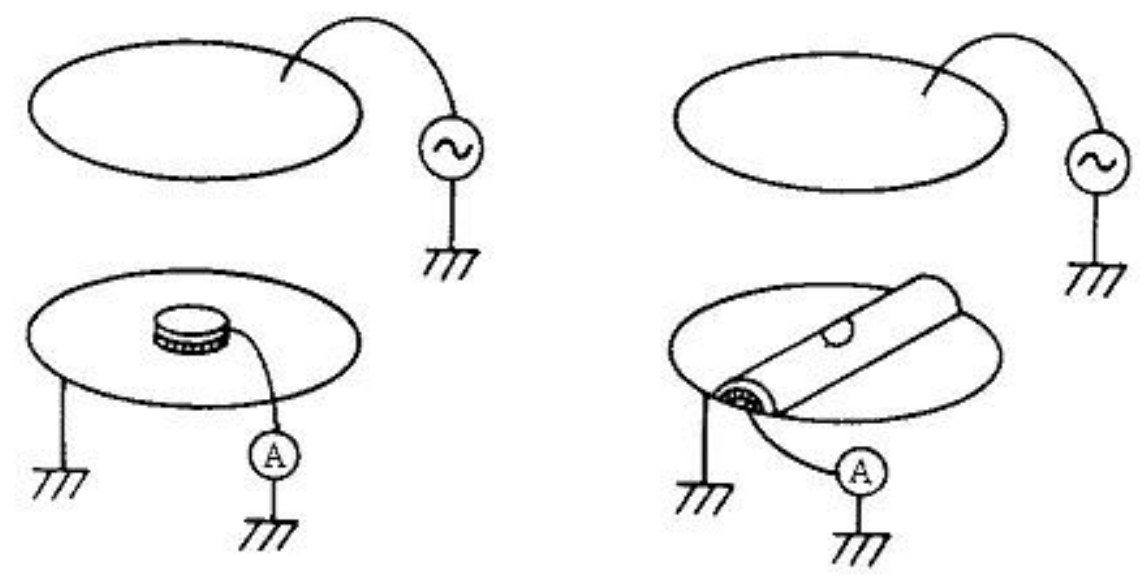

$\square$ conductor

Fig. 4. Schematic view of the experiments. (a) With a sensor. (b) With a cover.

(a)

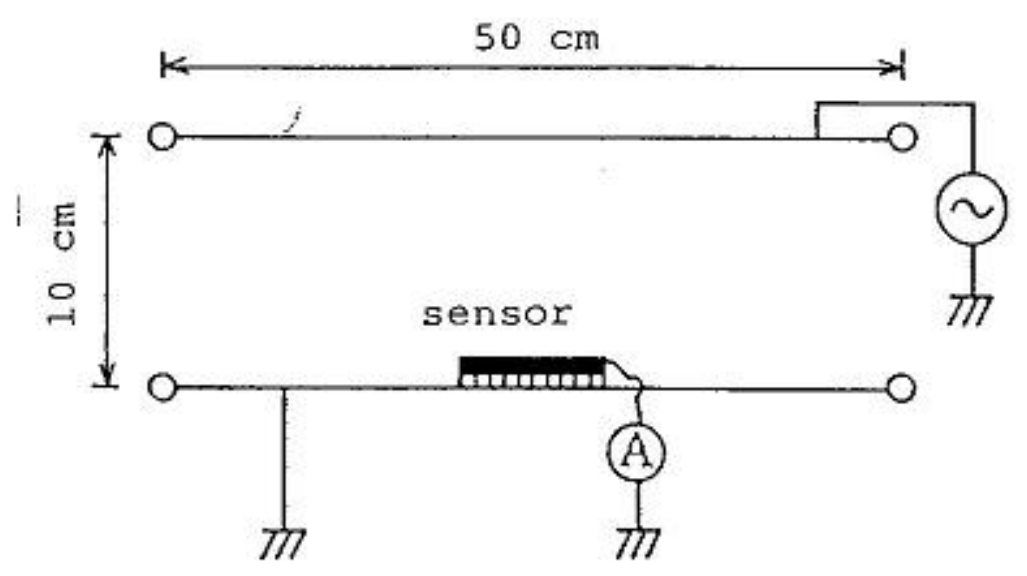

(b)

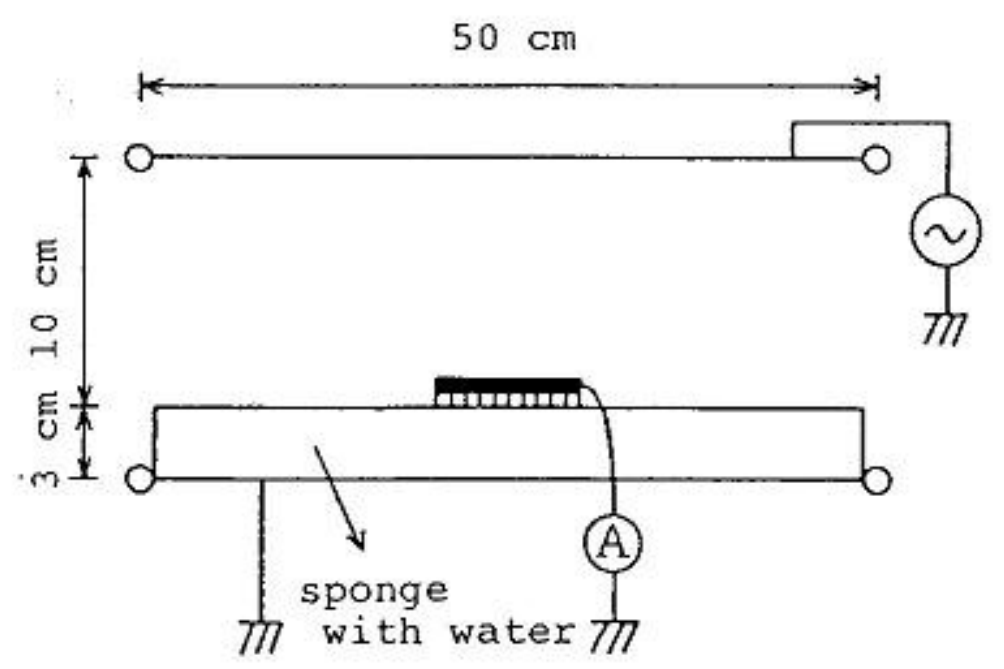

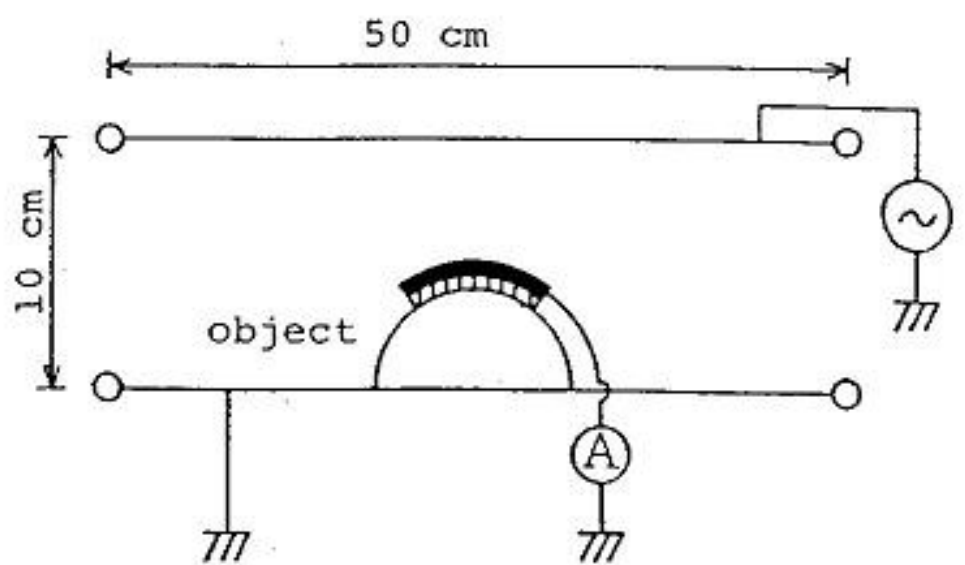

(c)

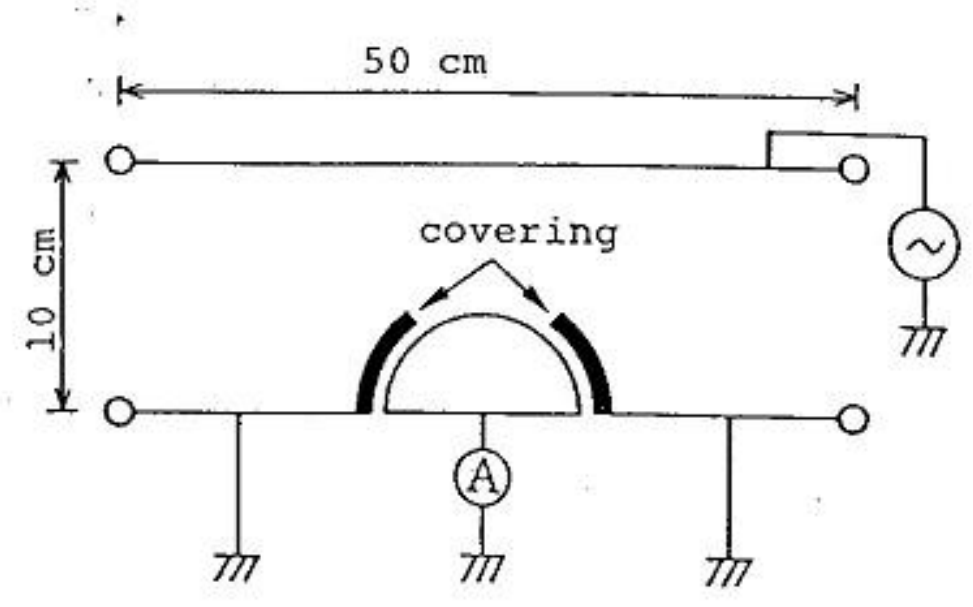

(d)

Fig. 5. Schematic front view of experiments. (a) Sensor on the lower electrode. (b) Sensor on the soft body (sponge with conductive water). (c) Sensor on the object surface. (d) Covering conductor on the object surface.

5(c)). 4) Using a cover instead of the flexible sensor, the curved surface of the half cylinder was covered with a conductive layer leaving the point of measurement uncovered (Fig. 5(d)). Since in the ELF range, a biological body is considered to be a conductor and a scaling effect in the size can be neglected [8], the above conditions are considered to represent the field measurement at the surface of a biological body in a uniform electric field such as that under transmission lines.

In the experiments, the current induced in the sensor (in cases 1), 2), and 3)), and in the object (in case 4)) were respectively measured, and the electric field was calculated according to (1) and (3). The current induced in the sensing part was fed to the following circuit through a shielded wire with a diameter of $2 \mathrm{~mm}$ and a length of about $0.5 \mathrm{~m}$. The distortion of the field caused by the wire was negligible small (less than 1 percent), since the diameter of the wire was small enough compared with the dimensions of the object. As the induced current is generally very small, it was amplified after the current-volt-

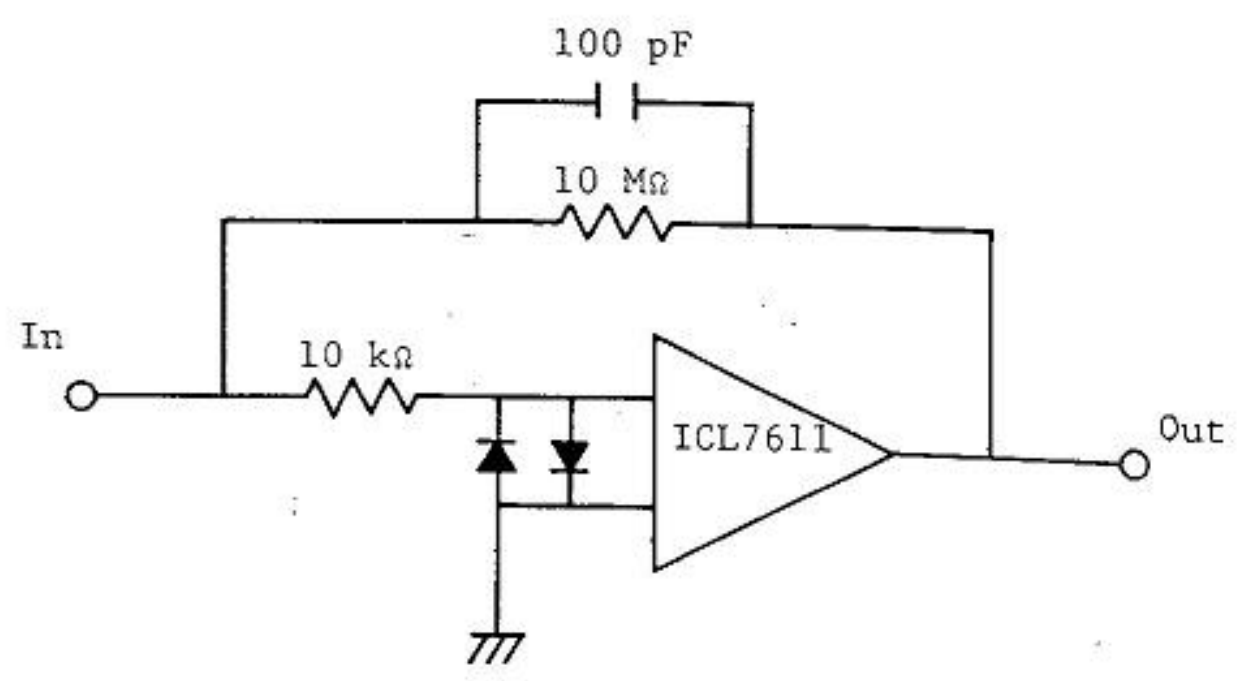

Fig. 6. The circuit for the current-voltage conversion.

age conversion. Fig. 6 shows the circuit for the currentvoltage conversion used in the measurements.

\section{Results And Discussion}

\section{A. Pressing Sensor}

A field sensor, which is usually disk-shaped and measures an induced current has a peripheral guard-ring to 


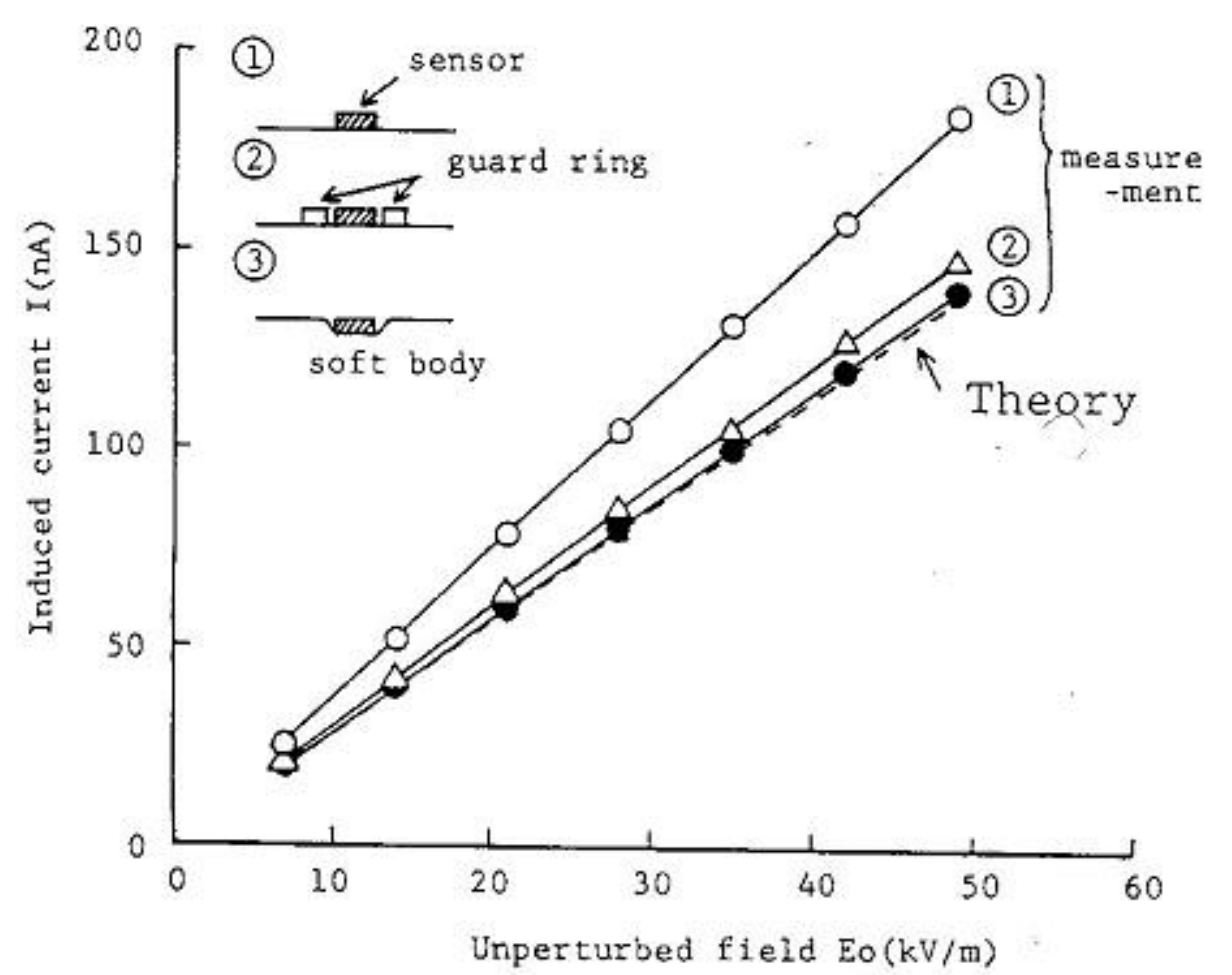

Fig. 7. Effect of pressing field sensor on soft surface. $\left(R=1: 8 \mathrm{~mm}, L_{G}\right.$ $=0.1 \mathrm{~mm}, W=2.0 \mathrm{~mm}, T=0.2 \mathrm{~mm}$.)

prevent unnecessary field concentration in the field sensing area of the sensor. With wider guard-rings, higher accuracy of the measurement is expected. According to our analysis, the width of the guard-ring has to be larger than the size of the sensing area to guarantee practical precision. Since the biological body surface is somewhat flexible, pressing the sensor down against the surface is one of the ways to increase the accuracy while keeping the size of the sensor small enough to assure good spatial resolution.

Fig. 7 shows the measured results of the field at the sensing area. They are for (1) a sensor without a guardring, (2) a sensor with a guard-ring, and (3) a pressed sensor without a guard-ring. The abscissa is the unperturbed field $E_{0}$ generated between the two circular plane parallel electrodes. The lower electrode was made of soft conductive material similar to that of a biological body. The ordinate is the current induced in the sensing area. The theoretical curve, that is, the ideal case with no perturbation is shown by the broken line in the figure. As expected, the results of (1) show a considerable error, while those of (2) show the effectiveness of the guard-ring. Case (3) shows better results than the sensor with the guard-ring, since it uses the peripheral soft body as a guard-ring and causes less perturbation to the field being measured.

After checking the effectiveness of this technique, the dependence of the accuracy in the measurement on the sensor thickness was analyzed. The determination of the thickness is important in practice to satisfy both the accuracy and the physical strength. Fig. 8 shows the calculated result of this dependence. The conditions of the sensor are illustrated in the figures in the upper left corner, namely the sensor without and with the guard-ring placed on the surface, and those pressed on the soft surface. The abscissa is the total sensor thickness $T$ and the ordinate is the field strength $E$ normalized to the unperturbed field $E_{0}$. In case (1), the error grows rapidly as the sensor thickness increases. As can be seen, the guard-ring suppresses this effect considerably, but still some error was left. Case (4) shows better characteristics than case

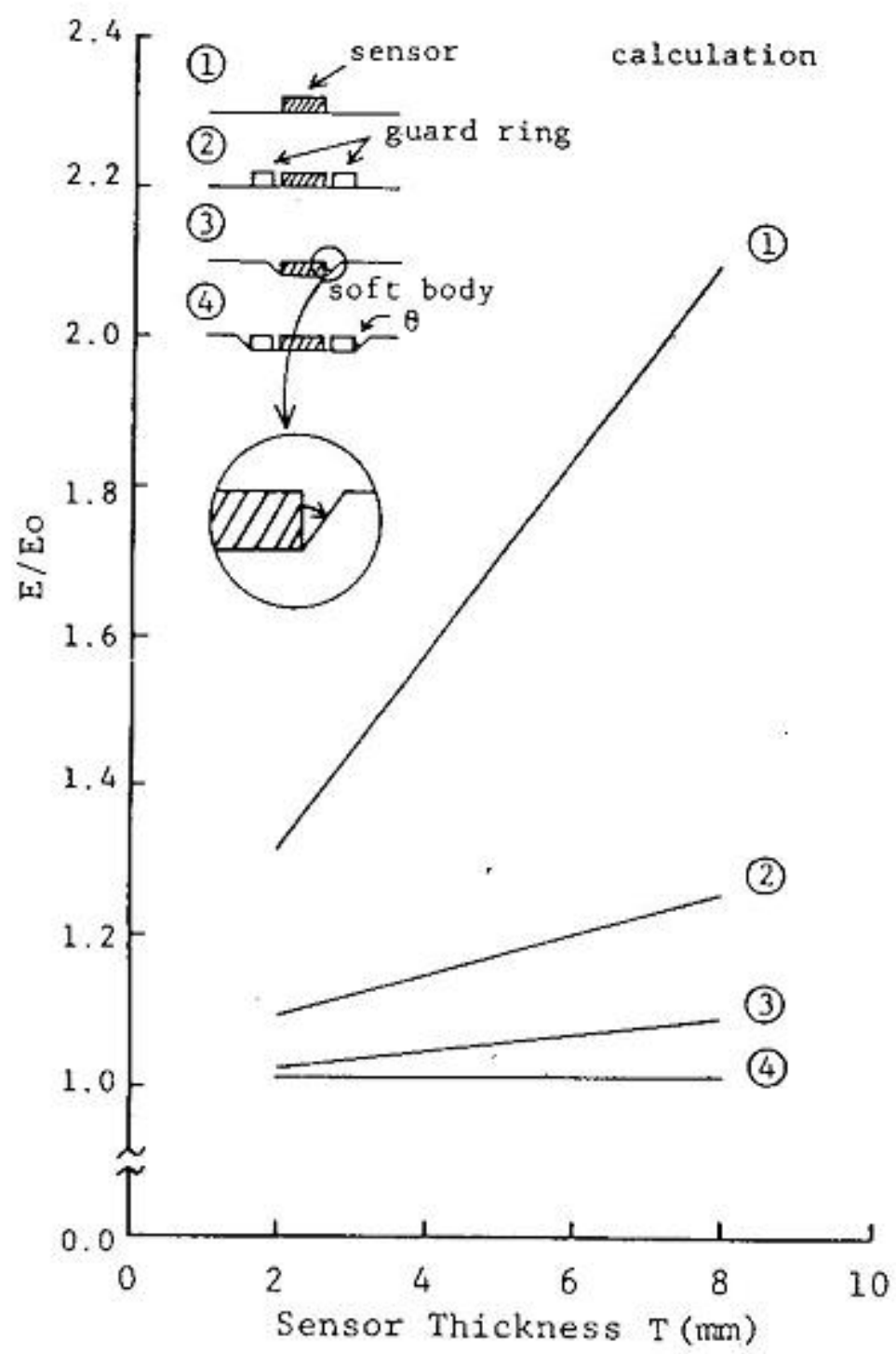

Fig. 8. Calculated electric field strength with different sensor thickness. $\theta$ is the angle between the edge of the sensor and the pressed soft body. $\left(R=1.8 \mathrm{~mm}, L_{G}=0.1 \mathrm{~mm}, W=2.0 \mathrm{~mm}, \theta=45^{\circ}\right.$.)

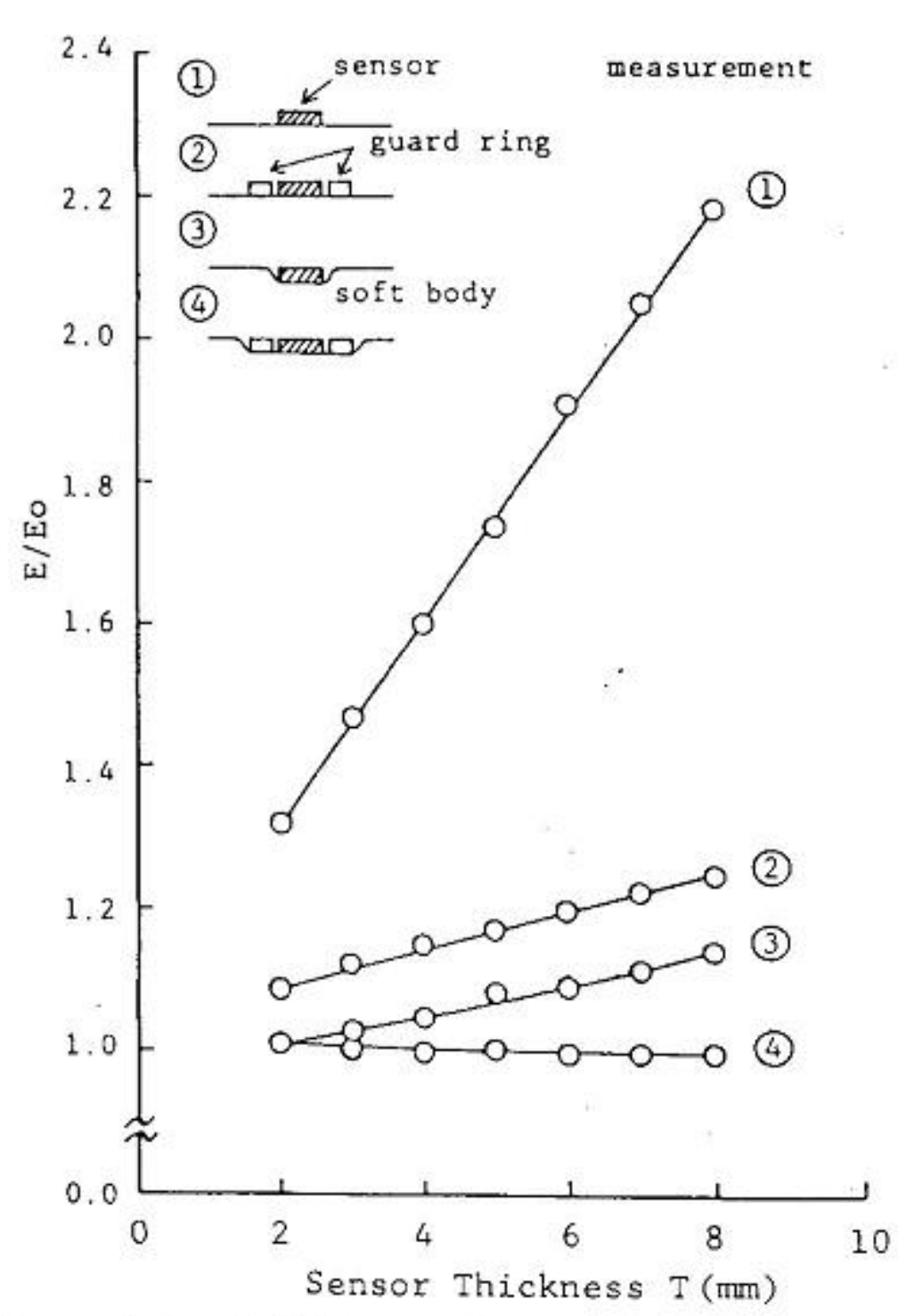

Fig. 9. Measured electric field strength with different sensor thickness. ( $R$ $=1.8 \mathrm{~mm}, L_{G}=0.1 \mathrm{~mm}, W=2.0 \mathrm{~mm}$.)

(3), since the gap between the sensor and the guard-ring is narrower than that between the sensor and the body. Although case (3) is not as good as case (4), this is adequate for most of the applications and has much better spatial resolution than case (4). These calculated results were verified by the experiment. Fig. 9 shows the results of the measurements corresponding to Fig. 8. They show 


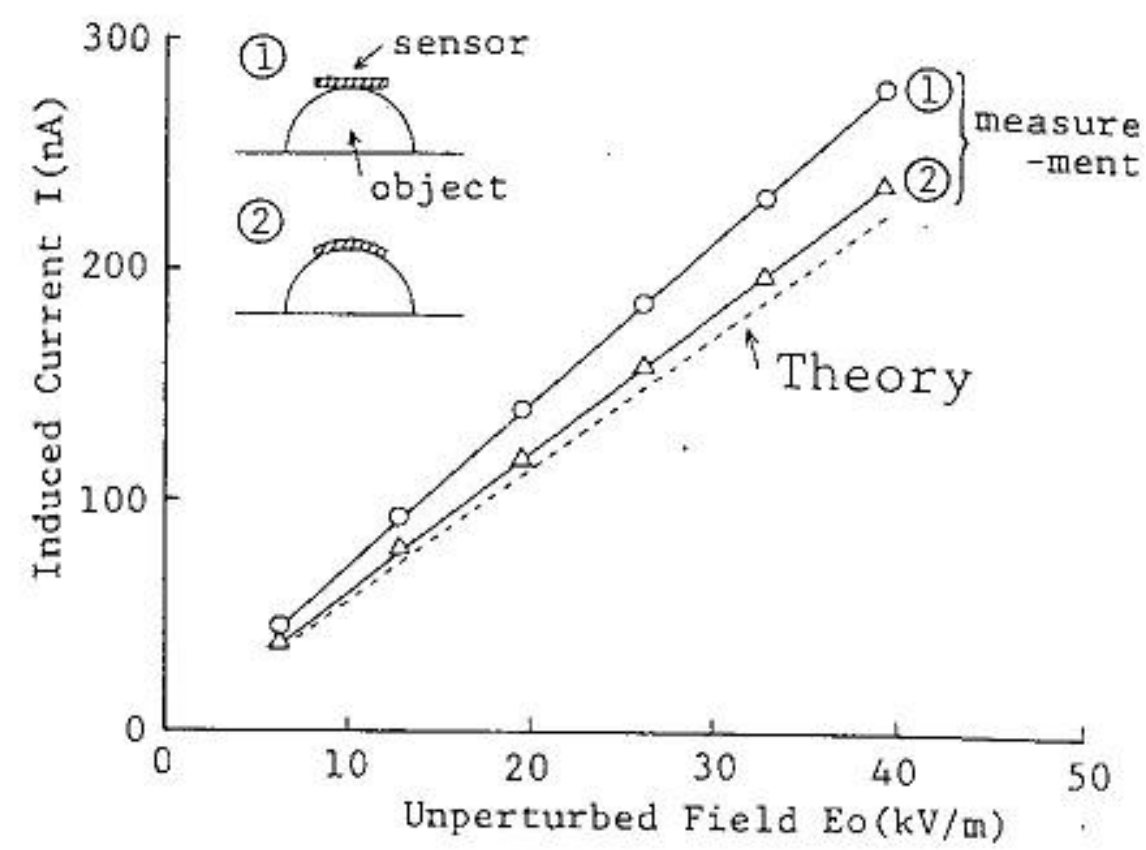

Fig. 10. Effect of using thin and flexible sensor on object surface. $(R=18 \mathrm{~mm}, T=0.13 \mathrm{~mm}$.)

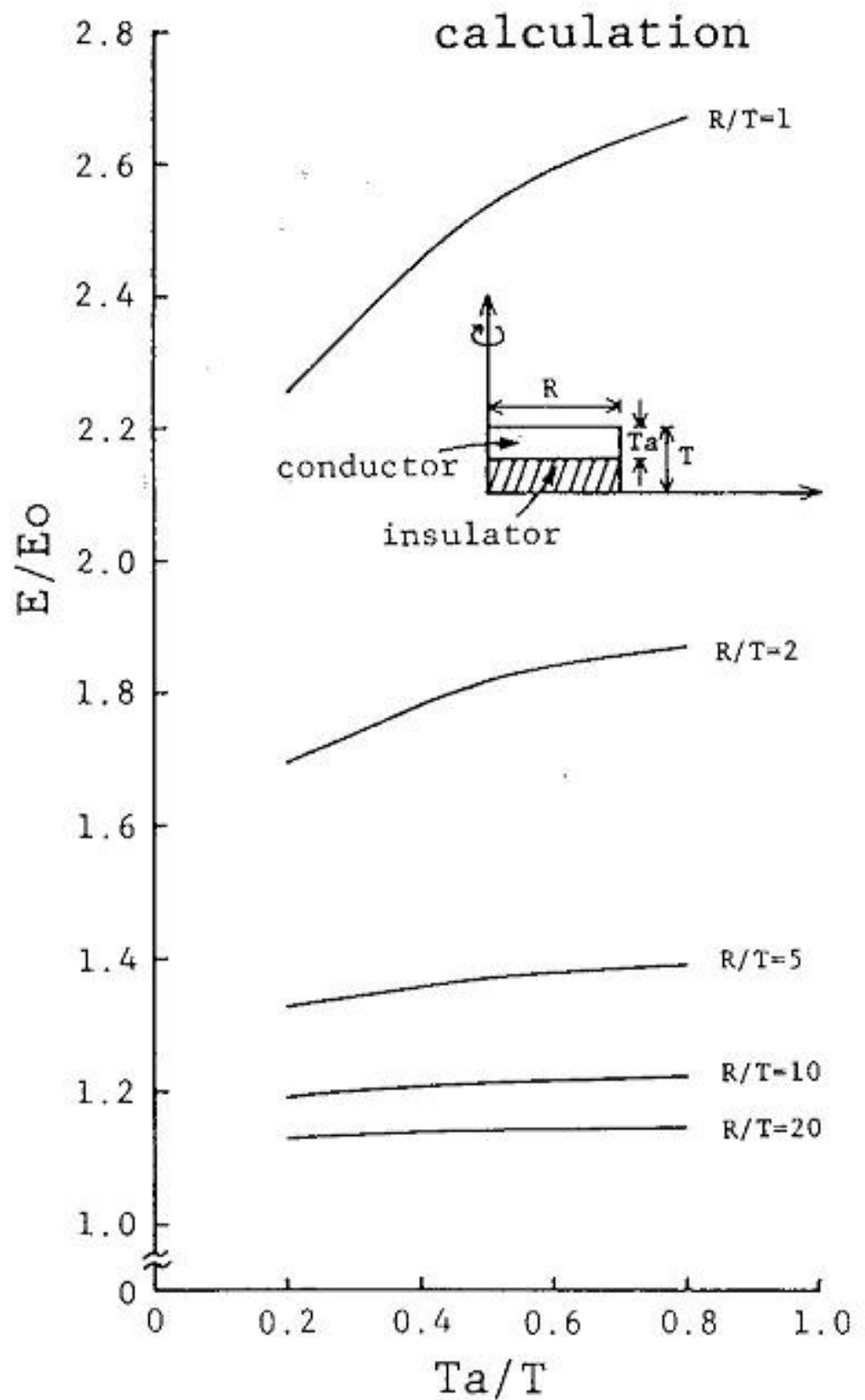

(a)

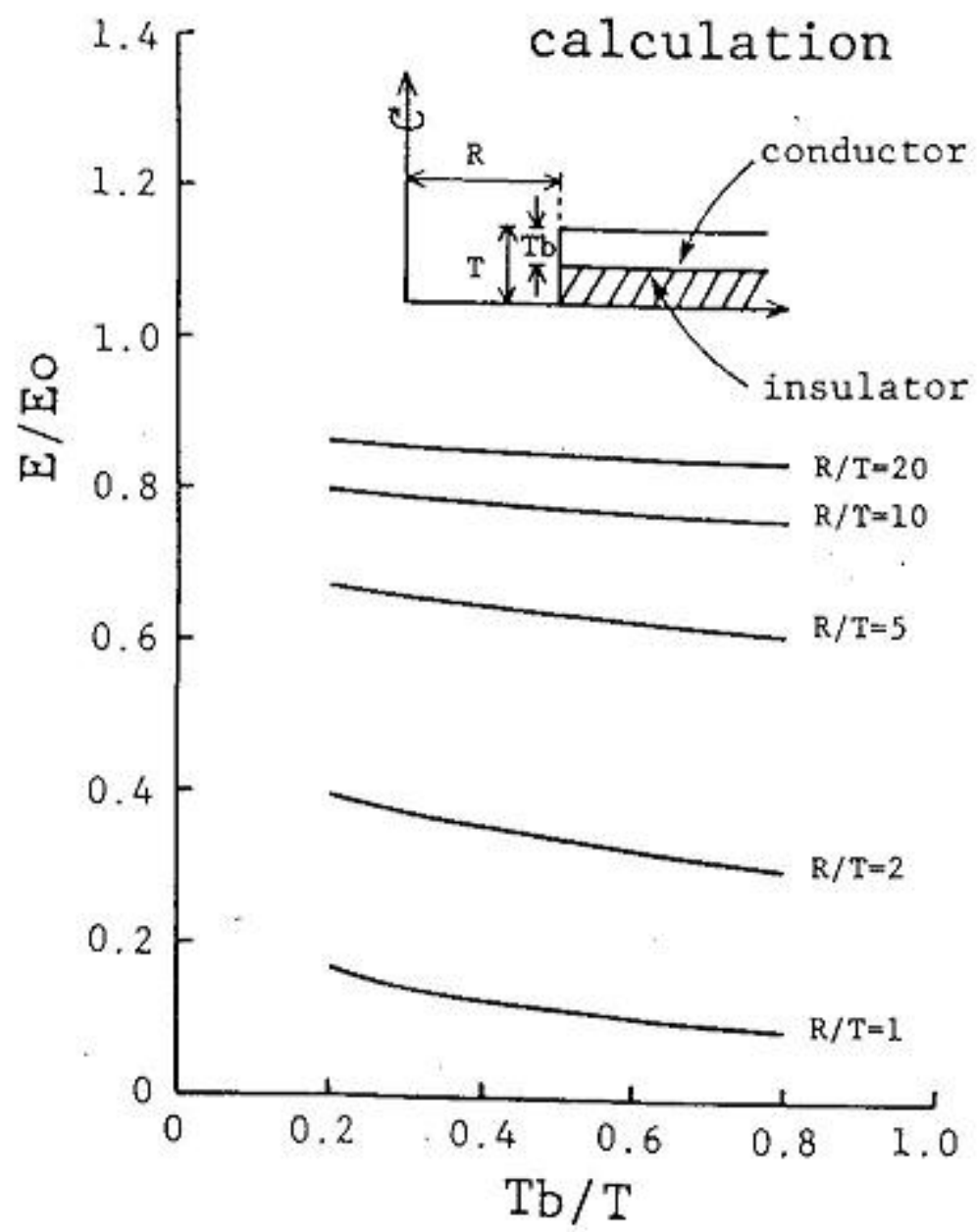

(b)

Fig. 11. Field strength with different conductor thickness. (a) With a sensor. (b) With covering conductor. ( $T_{a}, T_{b}=$ conductor thickness.).

good agreement with the calculated results qualitatively and quantitatively as well.

\section{B. The Thin Flexible Sensor}

The above technique is not applicable to the measurement of the hard parts of a human body, such as at the top of the head. For such an application, the usage of a thin flexible sensor without a guard-ring was considered. As a metallic sensor becomes thin, it becomes flexible and the accuracy of the measurement at a curved surface should be improved. Fig. 10 shows the results of the measurement at the conductive half cylinder placed on the lower electrode. The inserted figure (1) shows a disk-shape sensor placed on the cylinder, and the figure (2) shows the same sensor bent to fit the cylinder surface. The theoretical curve is obtained from (1) and (3). The ordinate is the current induced in the sensor. As can be seen in the figure, case (2) shows better results than case (1).

\section{Determination of Lower Limit}

In the above techniques, the sensor is generally protruding from the object surface and the measured values are always larger than a true field strength. Since the degree of the augmentation in the field strength varies depending upon the shape of the object, general calibration is difficult. Therefore, a technique was developed which gives a lower limit of the field strength. Using both techniques, the upper and lower limits of the field strength are 


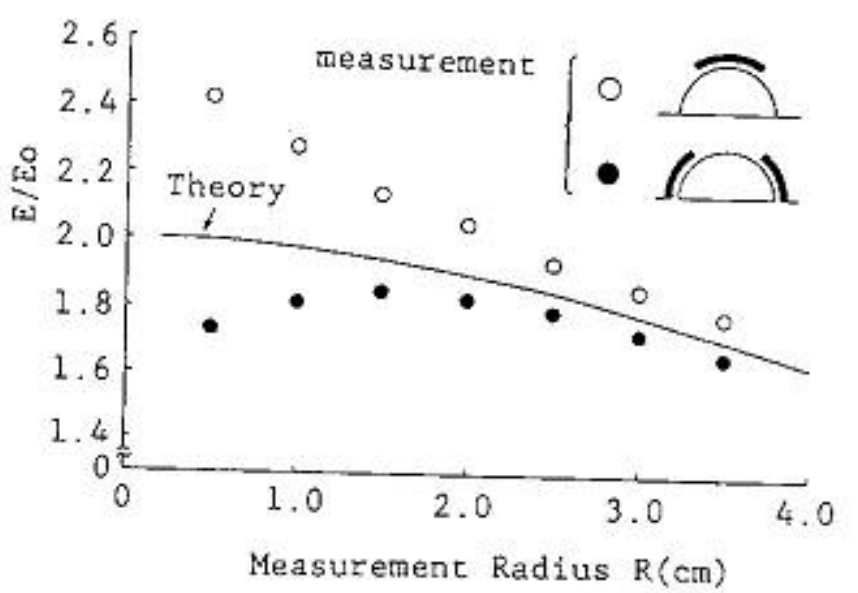

Fig. 12. Field strength at half-cylinder model surface: with a sensor (O), with covering conductor $(\bullet)$, (thickness of sensor and covering $=0.21$ $\mathrm{mm}$.

given, and a true value can be estimated within a known error boundary.

Fig. 11 shows the results of the calculation with the sensor and the cover placed on the ground plane as shown in Figs. 3(a) and (c). The abscissa is the conductor thicknesses $T_{a}$ (Fig. 11(a)) and $T_{b}$ (Fig. 11(b)) normalized by the total sensor thickness $T$, respectively. As can be seen, when $R / T$ is small, the error in the field strength becomes large as $T_{a}$ and $T_{b}$ become large. However, if $R / T$ is more than 10 , the field strength does not significantly depend on the conductor thicknesses, $T_{a}$ and $T_{b}$. Therefore, if the ratio $R / T$ is designed to be large enough, the measurement error should be small irrespective of the ratio of the conductor and the insulator thicknesses. With the sensor and the cover of more than $R / T=100$, an error of a few percent should be expected.

Fig. 12 shows the results of the measurement with the half cylinder model. They are with the flexible sensor placed on the measurement part of the curved object (O), and with the cover on the object surface leaving the measurement part uncovered $(\bullet)$. As can be seen, the error decreases as the radius $R$ of the measurement area becomes large. The theoretical value exists between the boundaries obtained in the two cases. In the above analysis, it was verified that the electric field at the surface of a biological body can be measured with a known error boundary.

\section{Conclusion}

In order to evaluate the field exposure to biological bodies more accurately, the characteristics of electric field measurement were investigated, and new techniques were proposed. They are pressing the sensor without a guardring against the soft surface of the body, using a thin flexible sensor along the curved surface of the body, and covering the body surface leaving the sensing part uncovered. According to the analysis, they improve the accuracy and the spatial resolution in the measurement of the electric field at a biological body surface. Their usefulness for the measurement of the electric field at the body surface has been shown. The first technique is simple to use and is useful for the mapping of the field distribution along soft parts of the body surface. The second technique enables us to measure the field at the spot where accurate measurement could not otherwise be expected. The third technique gives the lower limit of the true field and enables us to estimate the field strength with a known error boundary. Using these techniques it is possible to measure the field distribution over a biological body surface, and they provide useful tools for the investigation of biological effects of ELF electric fields.

\section{ACKNOWLEDGMENT}

The authors wish to thank Dr. H. Nakamura of the Central Institute of Electric Power Industry for his encouragement on this study, and Dr. T. Mikami and Dr. K. Yamamoto of Hokkaido University for their valuable ad vice.

\section{REFERENCES}

[1] E. J. Lerner, "Biological effects of electromagnetic fields," IEEE Spectrum, vol. 21, pp. 57-69, 1984.

[2] M. G. Morgan, H. K. Florig, I. Nair, and D. Lincoln, "Powerline fields and human health," IEEE Spectrum, vol. 22, pp. 62-68, 1985.

[3] E. L. Carstensen, Biological Effects of Transmission Line Fields. New York: Elsevier, 1987.

[4] WHO, "Extremely Low Frequency (ELF) Fields," Environmental Health Criteria 35, Geneva, 1984.

[5] C. J. Miller, "The measurement of electric fields in live line working," IEEE Trans. Power App. Syst., vol. PAS-86, pp. 493-498, 1967.

[6] D. W. Deno, "Currents induced in the human body by high voltage transmission line electric field measurement and calculation dose," IEEE Trans. Power App. Syst., vol. PAS-96, pp. 1517-1527, 1977.

[7] R. H. Galloway, H. M. Ryan, and M. F. Scott, "Calculation of electric field by digital computer," in Proc. Inst. Elect. Eng., vol. 114,
no. 6, pp. 824-829, 1967.

[8] W. T. Kaune and M. F. Gillis, "General properties of the interaction between animals and ELF electric field," Bioelectromagnetics, vol. 2, pp. $1-11,1981$ 